\title{
Efficacy of combined treatment with alendronate (ALN) and eldecalcitol, a new active vitamin D analog, compared to that of concomitant ALN, vitamin D plus calcium treatment in Japanese patients with primary osteoporosis
}

\author{
A. Sakai • M. Ito • T. Tomomitsu • H. Tsurukami • \\ S. Ikeda $\cdot$ F. Fukuda $\cdot$ H. Mizunuma $\cdot$ T. Inoue $\cdot$ H. Saito $\cdot$ \\ T. Nakamura $\cdot$ e-ADVANCED Study Group
}

Received: 6 October 2014 / Accepted: 5 December 2014 / Published online: 16 January 2015

(C) The Author(s) 2015. This article is published with open access at Springerlink.com

\begin{abstract}
Summary Combined treatment with alendronate and eldecalcitol was found to be more effective in reducing the bone turnover markers and increasing bone mineral density than alendronate treatment with vitamin D3 and calcium supplementation in the osteoporotic patients.

Introduction We compared the clinical efficacy and safety of combined treatment with alendronate plus eldecalcitol $($ ALN + ELD) with those of treatment with ALN plus vitamin D and calcium (ALN + VitD).

Methods Osteoporotic 219 patients were randomly assigned to the ALN + ELD, or the ALN + VitD group. Primary endpoint was the inter-group differences in lumbar spine BMD (L-BMD) at patient's last visit. Secondary endpoints
\end{abstract}

e-ADVANCED Study = Eldecalcitol; Alendronate plus vitamin D Vs. AleNdronate Combined with ElDecalcitol Study

A. Sakai

Department of Orthopaedic Surgery, University of Occupational and Environmental Health, Kitakyushu, Japan

M. Ito

Medical Work-Life Balance Center, Nagasaki University Hospital,

Nagasaki, Japan

T. Tomomitsu

Department of Radiological Technology, Kawasaki College of Allied

Health Professions, Kurashiki, Japan

H. Tsurukami

Tsurukami Clinic of Orthopaedic and Rheumatology Hospital,

Kumamoto, Japan

S. Ikeda

Ken-Ai Memorial Hospital, Onga, Japan included the differences in BMD at other sites and the bone turnover marker (BTM) levels.

Results L-BMD, total hip BMD and femoral neck (FN-BMD) increased from baseline by 7.30, 2.41, and $2.70 \%$ in the ALN + ELD group, and by 6.52, 2.27, and $1.18 \%$ in the ALN + VitD group, respectively. Inter-group differences of the LBMD and total hip BMD values were not significant. The increase of the FN-BMD was larger in the ALN + ELD group than the ALN + VitD group. Reductions of the BTMs were greater in the ALN + ELD group than the ALN + VitD group. Interaction of the percent increase of the L-BMD with the baseline values of the BTMs was observed in the ALN + VitD group only. The increases of the FN-BMD in patients with lower baseline values of type-I-collagen C-telopeptide
F. Fukuda

Kitakyushu General Hospital, Kitakyushu, Japan

H. Mizunuma

Department of Obstetrics and Gynecology, Hirosaki University

School of Medicine, Hirosaki, Japan

T. Inoue

Taisho Pharmaceutical Co. Ltd, Tokyo, Japan

H. Saito

Chugai Pharmaceutical Co. Ltd, Tokyo, Japan

T. Nakamura $(\square)$

National Center for Global Health and Medicine, 1-21-1 Toyama,

Shinjyuku-ku, Tokyo 162-8655, Japan

e-mail: t-nak@utopia.ocn.ne.jp 
(sCTX) and serum $25(\mathrm{OH})$ D levels $<20 \mathrm{ng} / \mathrm{mL}$ were significantly larger in the ALN + ELD group than the other group. Conclusion Combination treatment of ALN plus ELD was more effective in reducing the BTMs and increasing the FNBMD than ALN treatment with vitamin D3 and calcium.

Keywords Bisphosphonate $\cdot$ Bone mineral density . Bone turnover markers $\cdot$ Eldecalcitol $\cdot$ Vitamin D

\section{Introduction}

Eldecalcitol (ELD) is a newly developed active vitamin D analog, bearing a hydroxypropyloxy substituent at the $2 \beta$ position of $1 \alpha, 25(\mathrm{OH})_{2} \mathrm{D}_{3}$ (calcitriol) [1]. In a randomized, placebo-controlled, double-blind clinical trial conducted in patients with osteoporosis receiving vitamin D supplementation, 48-week treatment with ELD increased the lumbar spine and total hip bone mineral density (BMD) in a dose-dependent manner without causing sustained hypercalcemia [2]. In a fracture prevention trial, 3-year treatment with ELD significantly reduced the incidence of vertebral and wrist fractures to the greater extent than treatment with alfacalcidol, which is $1 \alpha$-hydroxyvitamin $\mathrm{D}_{3}$; the incidence of adverse events during the trial was similar between the two treatment groups [3]. Based on these results, ELD has been approved for the treatment of osteoporosis in Japan.

Drugs of the bisphosphonate (BP) group have been used as potent therapeutic agents in the treatment of osteoporosis $[4,5]$. BPs increase the BMD and suppress bone turnover by inhibiting osteoclastic activity and inducing apoptosis of osteoclasts. They bind strongly to the bone and remain in the bone for prolonged periods of time. Serum calcium $(\mathrm{Ca})$ decreases slightly during $\mathrm{BP}$ treatment due to a reduction of the $\mathrm{Ca}$ efflux from the bone. Because even subtle reduction of the serum $\mathrm{Ca}$ causes an increase in the secretion of parathyroid hormone (PTH), which enhances bone turnover, the $\mathrm{Ca}$ balance in the body becomes negative if BPtreated patients have vitamin D insufficiency or deficiency. Therefore, it is generally accepted that during the treatment of osteoporosis with BPs, vitamin D and $\mathrm{Ca}$ supplementation is necessary to enhance the efficacy of the BP treatment and prevent negative $\mathrm{Ca}$ balance in the body. We hypothesized that the addition of active vitamin D in place of vitamin D plus Ca to BP therapy may be more effective to increase the BMD and regulate the bone turnover. In this study, we compared the clinical efficacy and safety of addition of ELD in comparison to that of plain vitamin $\mathrm{D}$ plus $\mathrm{Ca}$ in patients with primary osteoporosis receiving alendronate (ALN) treatment.

\section{Subjects and methods}

All procedures performed in studies involving human participants were in accordance with the ethical standards of the institutional and/or national research committee and with the 1964 Helsinki declaration and its later amendments or comparable ethical standards.

Study design and patients

The e-ADVANCED (Eldecalcitol; Alendronate plus vitamin D Versus AleNdronate Combined with ElDecalcitol) study was a randomized, unblinded clinical trial carried out to assess the efficacy on BMD, bone turnover markers (BTMs) and safety, of addition of ELD as compared to that of vitamin D plus $\mathrm{Ca}$ supplementation in osteoporotic patients receiving weekly ALN treatment (JapicCTI-No.111653). We analyzed the data of 219 subjects, including 214 females and 5 males, ranging in age from 60 to 90 years (yr.) (mean, $71.5 \mathrm{yr}$.), who had osteoporosis (defined as a low BMD (70 \% of the young adult mean (YAM); $0.708 \mathrm{~g} / \mathrm{cm}^{2}$ by the Hologic QDR series) or osteopenia ( $80 \%$ of the YAM; $0.809 \mathrm{~g} / \mathrm{cm}^{2}$ by the Hologic QDR series) with at least one vertebral fracture, according to the criteria of the Japanese Society for Bone and Mineral Research [6]. Prevalent vertebral fractures were assessed by $\mathrm{x}$-ray examination of the vertebrae, and diagnosed according to the criteria of the Japanese Society for Bone and Mineral Research [7]. All the subjects were 60 years old or older, and patients were excluded from the study if they had fractures in any of the lumbar vertebrae L2-L4 or had disorders such as primary hyperparathyroidism, Cushing's syndrome, gonadal insufficiency, poorly controlled diabetes mellitus, or other causes of secondary osteoporosis, or a history of/suspected active urolithiasis at any time. Subjects were also excluded if they had taken BPs within 48 weeks before entry into this study, taken glucocorticoids, calcitonin, vitamin K, active vitamin $\mathrm{D}$ compounds, ipriflavones, selective estrogen receptor modulators, or hormone replacement therapy within 8 weeks prior to entry into this study, or taken PTH, antireceptor activator of NF-kappaB ligand (RANKL) antibody, cathepsin $\mathrm{K}$ inhibitor prior to entry, had serum calcium levels above or below the normal range, serum creatinine levels of over $1.0 \mathrm{mg} / \mathrm{dL}$, any disorder associated with delayed passage of food through the esophagus, received/were scheduled to receive any invasive dental procedures, clinically significant hepatic or cardiac disorders, or active malignant tumor or prior therapy for malignancy within the previous 3 years. The subjects were enrolled at 13 centers in Japan. The study was conducted with the approval of the institutional review board at each center and in accordance with the Declaration of Helsinki and the Japanese of Good Clinical Practice Guidelines. Written informed consent was obtained from each patient prior to any study-related procedure. 


\section{Treatment}

Patients were randomly assigned at a 1:1 ratio to receive oral ALN at the dose of $35 \mathrm{mg}$ weekly, the approved dosage in Japan, plus oral ELD at the dose of $0.75 \mu \mathrm{g}$ daily (ALN + ELD group), or oral ALN at the dose of $35 \mathrm{mg}$ weekly with oral vitamin D $400 \mathrm{IU}+\mathrm{Ca} 610 \mathrm{mg}$ daily (ALN + VitD group). Randomization was performed by a computerized system. The subjects were stratified into groups on the basis of the L2-4 BMD values ( $\geq 70 \%$ YAM $/<70 \%$ YAM). The randomization sequence was created by the person responsible for the investigational product randomization. Neither the patients nor the investigators were blinded to the treatment assignment at any time during the study period. Compliance with the study treatment was assessed based on the medication diaries maintained by each subject.

\section{Assessments}

The BMD values at the lumbar spine (L-BMD), femoral neck (FN-BMD) and total hip in the posterior-anterior projections were measured at the baseline and at 24 and 48 weeks by dualenergy x-ray absorptiometry (DXA). The 13 study centers participating in this trial were all equipped with the Hologic QDR series (QDR2000, QDR4500, Explorer, Delphi or Discovery, Hologic, Inc.) for the BMD measurements. A central facility (Department of Radiological Technology, Kawasaki College of Allied Health Professions) performed quality assurance of the longitudinal adjustment. Adjustment for DXA machine differences was made to calibrate each machine with standardized phantoms. All the DXA measurements were analyzed at a central site by a radiologist who was blinded to the treatment group assignment. Radiographs of the thoracic and lumbar spine were taken at the baseline and at 48 weeks to assess the incidence of vertebral fractures. To identify morphometric vertebral fractures, the vertebral bodies in the lateral projections from Th4 to L4 were quantitated using a semi-quantitative (SQ) methodology [8] by a central committee who was also blinded to the treatment assessment. Patients with more than one incident L2-L4 vertebral fracture during the treatment period were excluded from the BMD analysis. Hip BMD images not satisfying the appropriate positioning criteria were also excluded by the central committee.

Serum and postprandial urine samples were collected the baseline and at 4, 12, 24, and 48 weeks of treatment to evaluate Ca-related parameters such as the serum $\mathrm{Ca}$, serum phosphorus, serum albumin, serum creatinine, and urinary $\mathrm{Ca}$ adjusted by $\mathrm{mg} / \mathrm{dL}$ GF. In addition, routine blood biochemical analyses, including evaluation of the hematological, hepatic, and renal functions were conducted at the baseline and at 24 and 48 weeks. Bone turnover markers, including type I
Table 1 Baseline characteristics of the enrolled patients

\begin{tabular}{lll}
\hline & $\begin{array}{l}\text { ALN + ELD group } \\
(n=110)\end{array}$ & $\begin{array}{l}\text { ALN + VitD group } \\
(n=109)\end{array}$ \\
\hline Age (years) & $71.5 \pm 7.3$ & $71.6 \pm 6.6$ \\
Gender (female/male) & $109 / 1$ & $105 / 4$ \\
Height $(\mathrm{cm})$ & $150.0 \pm 5.9$ & $149.8 \pm 6.3$ \\
BMI $\left(\mathrm{kg} / \mathrm{m}^{2}\right)$ & $22.3 \pm 3.1$ & $21.7 \pm 2.9$ \\
Number of prevalent vertebral fractures & & \\
0 & $54(50.9 \%)$ & $37(36.6 \%)$ \\
1 & $34(32.1 \%)$ & $41(40.6 \%)$ \\
$\geq 2$ & $16(15.1 \%)$ & $21(20.8 \%)$ \\
LS-BMD $\left(\mathrm{mg} / \mathrm{cm}^{2}\right)$ & $653 \pm 77$ & $655 \pm 90$ \\
Total hip BMD $\left(\mathrm{mg} / \mathrm{cm}^{2}\right)$ & $666 \pm 82$ & $659 \pm 95$ \\
FN-BMD $\left(\mathrm{mg} / \mathrm{cm}^{2}\right)$ & $518 \pm 70$ & $518 \pm 84$ \\
sCa $(\mathrm{mg} / \mathrm{dL})$ & $9.43 \pm 0.32$ & $9.46 \pm 0.34$ \\
uCa $(\mathrm{mg} / \mathrm{dL} \mathrm{GF})$ & $0.099 \pm 0.073$ & $0.103 \pm 0.067$ \\
BAP $(\mu \mathrm{g} / \mathrm{L})$ & $19.6 \pm 7.6$ & $18.6 \pm 7.4$ \\
P1NP $(\mathrm{ng} / \mathrm{mL})$ & $54.8 \pm 22.2$ & $57.1 \pm 26.9$ \\
TRACP-5b $(\mathrm{mU} / \mathrm{dL})$ & $494 \pm 160$ & $526 \pm 193$ \\
uNTX $(\mathrm{nmol} \mathrm{BCE} / \mathrm{mmol} \mathrm{Cr})_{\mathrm{sCTX}(\mathrm{pmol} / \mathrm{mL})}^{68.2 \pm 27.3}$ & $70.0 \pm 30.2$ \\
$25(\mathrm{OH}) \mathrm{D}(\mathrm{ng} / \mathrm{mL})$ & $4.30 \pm 2.09$ & $4.55 \pm 2.33$ \\
$1,25(\mathrm{OH})_{2} \mathrm{D}(\mathrm{pg} / \mathrm{mL})$ & $18.7 \pm 6.2$ & $19.2 \pm 6.7$ \\
Intact-PTH $(\mathrm{pg} / \mathrm{mL})$ & $62.4 \pm 26.8$ & $58.6 \pm 25.7$ \\
\hline & $50.4 \pm 17.6$ & $50.8 \pm 15.2$ \\
\hline
\end{tabular}


collagen C-telopeptide (sCTX) ( $\beta$-Crosslaps N, Fujirebio Inc, Tokyo), urinary type I collagen N-telopeptide (uNTX) (Osteomark NTx; Alere, Orlando, FL), tartrate-resistant acid phosphatase 5b (TRACP-5b) (Osteolinks TRAP-5b, DS Pharma Biomedical Co., Ltd, Osaka), serum bone-specific alkaline phosphatase (BAP) (Access Ostase, Beckman Coulter, Inc., Brea, CA), and amino-terminal propeptide of type 1 procollagen (P1NP) (Procollagen Intact P1NP, TFB Inc. Tokyo) were assessed at the baseline and at 12, 24, and 48 weeks. Blood levels of the calcium-regulating hormones such as intact PTH (Intact-PTH, Roche Diagnostics, Hoffmann-La Roche Ltd, Indianapolis, IN), 25(OH) D (CPBA, LSI Medience Corporation, Tokyo), and 1, $25(\mathrm{OH})_{2} \mathrm{D}(1,25(\mathrm{OH}) 2 \mathrm{D}$ RIA Kit [TFB], Fujirebio Inc., Tokyo) were also evaluated at the baseline and at 24 and 48 weeks.

All the patients were asked about the occurrence of any adverse events at each visit, and all adverse events were analyzed, regardless of the investigators' assessments of the causality. The Medical Dictionary for Regulatory Activities (MedDRA, version 16.1) was used to categorize the reported adverse events.

\section{Statistical analyses}

The primary endpoint was the percent change of the lumbar spine BMD (L-BMD) at the patient's last visit, week 48 from the baseline. Analyses were performed according to the intent-totreat principle, in which all randomized patients who had taken at least one dose of the study drug and had undergone both a baseline and at least one post-randomization measurement of the BMD, bone markers, and other parameters were included. Missing data were imputed by the last observation carried forward (LOCF) method. ANCOVA adjusted for the baseline data was used between the ALN + ELD and ALN + VitD groups. Significance level is used $5 \%$ for two-sided $p$ value. As exploratory analysis, group means and $95 \%$ confidence intervals are calculated for the percent changes from the baseline in the lumbar, total hip and femoral neck BMD, and were used to assess the changes within each group for each visit. The relationships between the variables were assessed by calculation of the Pearson's correlation. Multiplicities adjustments are not used for $p$ values of exploratory analysis.

\section{Results}

Patient distribution and baseline characteristics

A total of 230 patients were enrolled, of which 11 patients did not receive any treatment; the remaining 219 patients were included in the full analysis set (FAS). The patients were randomized to the ALN + ELD $(n=110)$ or ALN + VitD $(n=109)$ group. The study was completed as per protocol in 196 patients (PPS; 101 patients in the ALN + ELD group and 95 patients in the ALN + VitD group). The baseline characteristics were well-balanced between the two treatment groups (Table 1). The mean serum 25(OH) D level at the baseline was $18.7 \mathrm{ng} / \mathrm{mL}$ ( $66.7 \%$ of the subjects with values $<20 \mathrm{ng} / \mathrm{mL})$ in the ALN + ELD group, and $19.2 \mathrm{ng} / \mathrm{mL}(61.4 \%$ of the subjects with values $<20 \mathrm{ng} / \mathrm{mL}$ ) in the ALN + VitD group.

Bone mineral density and BTMs

The L-BMD, total hip BMD, and FN-BMD increased from baseline significantly by $7.30,2.41$, and $2.70 \%$, on average, in the ALN + ELD group, and by $6.52,2.27$, and $1.18 \%$ in the

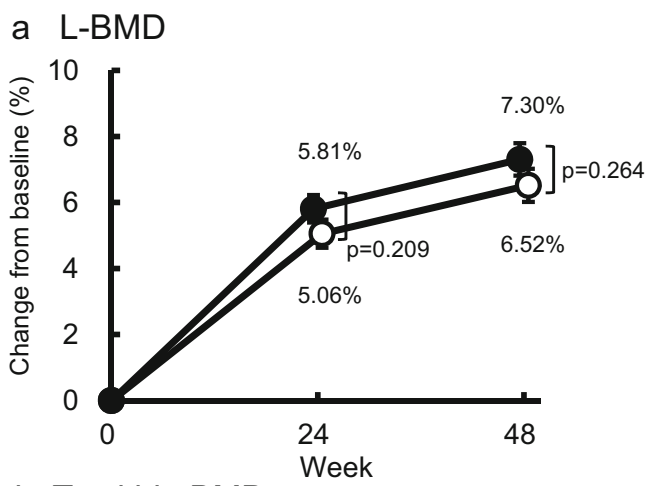

b Total hip BMD
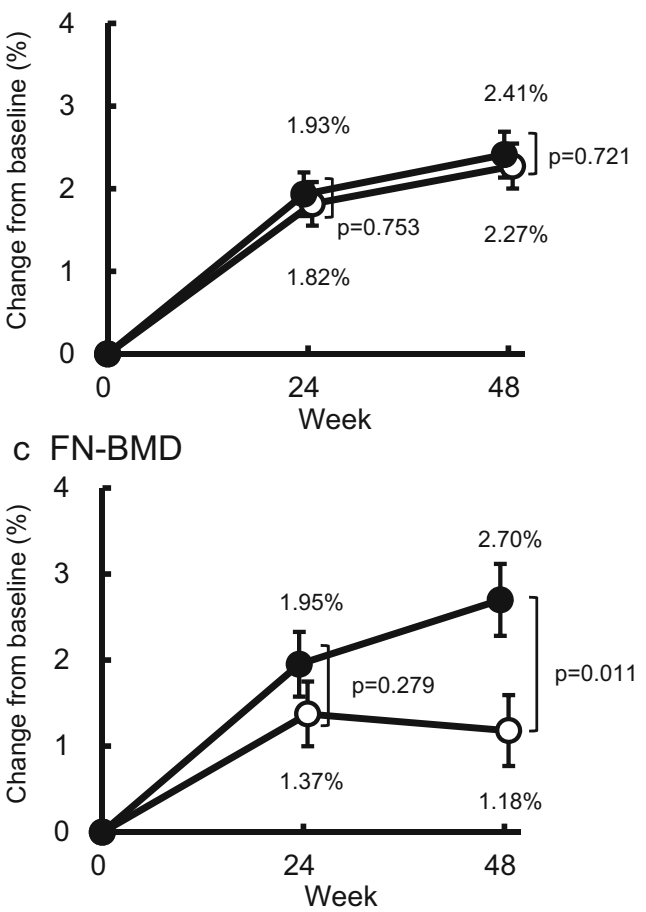

Fig. 1 Percent change from the baseline of the a lumbar spine BMD, b total hip BMD, and $\mathbf{c}$ femoral neck BMD. Symbols indicate the groups as follows: ALN + ELD, closed circles $(\bullet)$; ALN + VitD, open circles $(\circ)$. Data are represented as least squares (LS) mean \pm standard error (SE) 
Fig. 2 Percent changes from the baseline of the levels of a sCTX, b uNTX, c TRAC-5b, d BAP, and e P1NP. Symbols indicate the groups as follows: ALN + ELD, closed circles $(\bullet)$; ALN + VitD, open circles $(\circ)$. Data are represented as LS mean $\pm \mathrm{SE}$

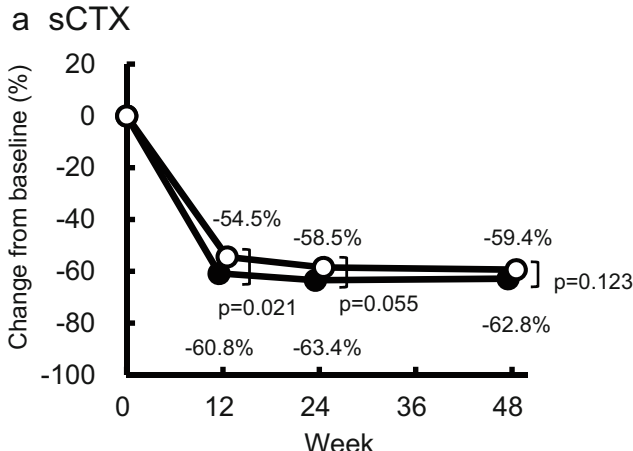

b UNTX
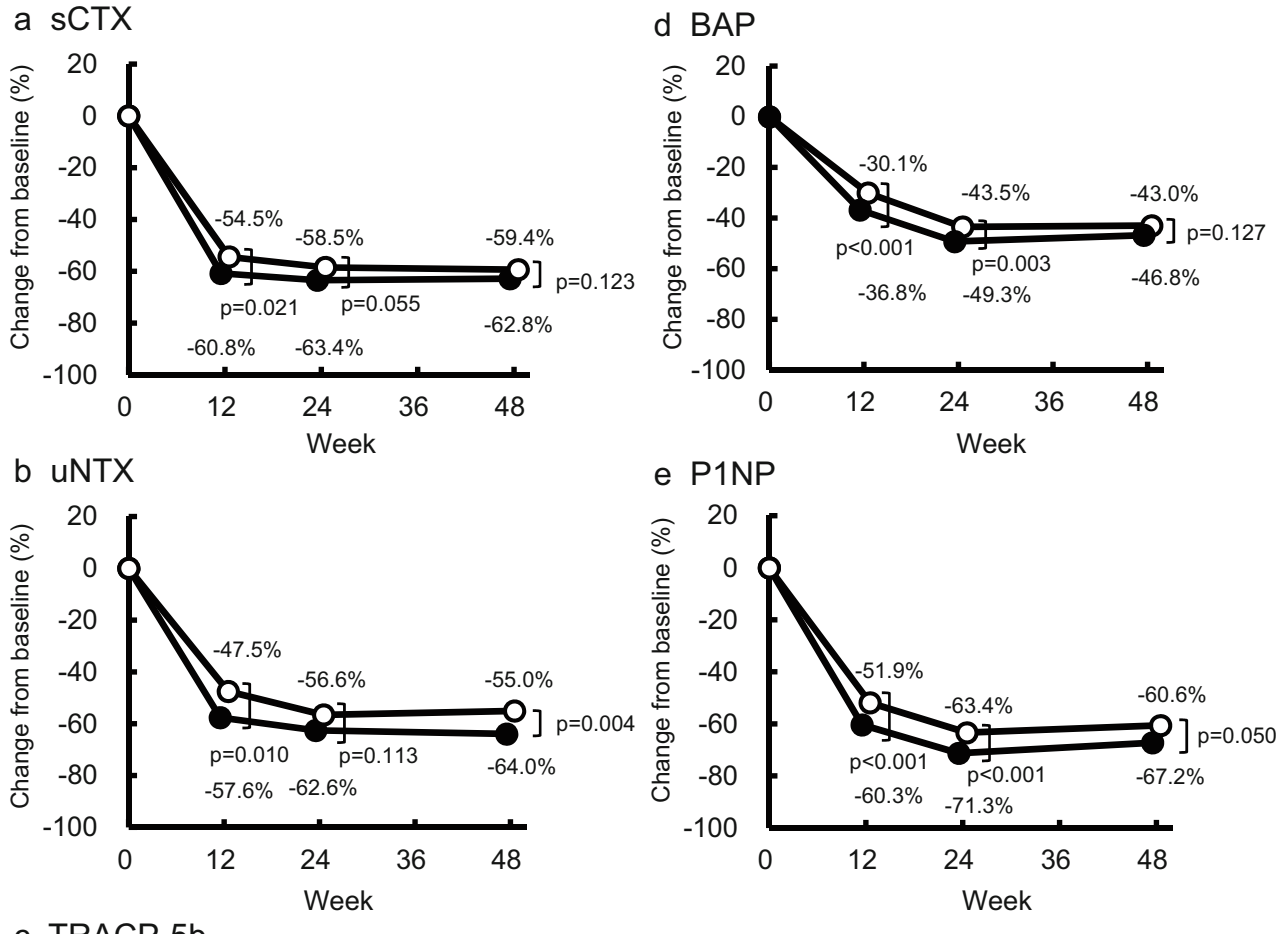

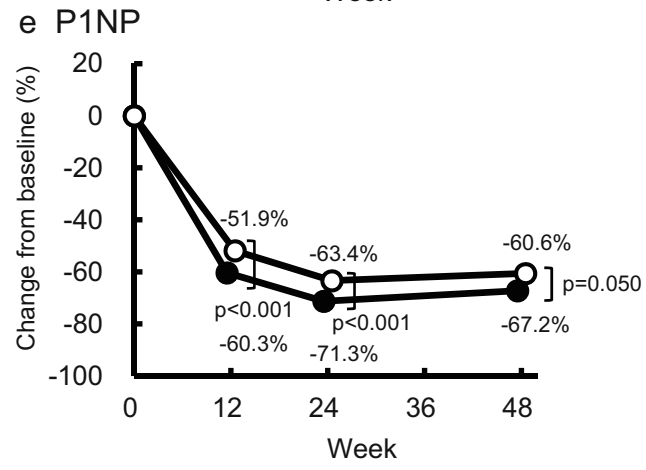

c TRACP-5b

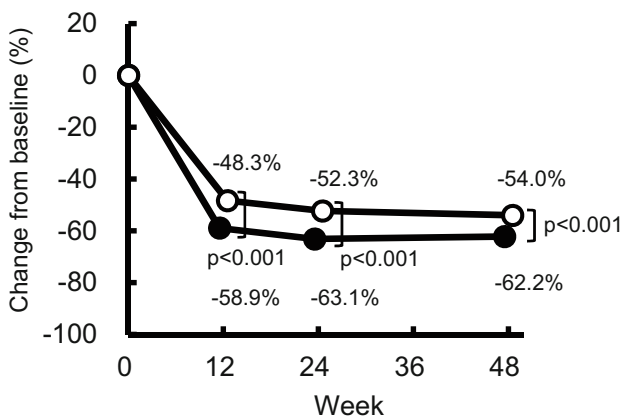

ALN + VitD group, respectively, at 48 weeks (Fig. 1a-c). Inter-group differences in the L-BMD and total hip BMD values were not significant. The percent increases in the FNBMD were markedly larger in the ALN + ELD group than those in the ALN + VitD group at 48 weeks $(p=0.011)$.

Substantial decrease in the levels of all BTMs (sCTX, uNTX, TRACP-5b, BAP, and P1NP) was observed from 12 weeks onward in both treatment groups, as compared to the respective baseline levels (Fig. 2a-e). The changes from the baseline of all the BTMs were larger in the ALN + ELD group at 12 weeks onwards than the corresponding changes in the ALN + VitD group.

BMD changes in relation to the baseline values of the BTMs and serum $25(\mathrm{OH}) \mathrm{D}$

Pearson's correlation analyses revealed that the percent increase of the L-BMD was strongly correlated with the baseline levels of all the BTMs in the ALN + VitD group (Table 2), but not in the ALN + ELD group. Then, patients were stratified into tertiles by the baseline levels of the BTMs. In the ALN + VitD group, the percent increase of the L-BMD was larger in the high BTM tertile and smaller in the low BTM tertile (Fig. 3a-e). In the ALN + ELD group, however, the percent BMD increase did not depend on the baseline levels of the BTMs. The percent increases of the L-BMD in patients with low tertile levels of sCTX, uNTX, and BAP, respectively,

Table 2 Correlations between the percent change of the lumbar spine $\mathrm{BMD}$ and the baseline values of the BTMs

ALN + ELD group $(n=105) \quad$ ALN $+\operatorname{VitD}$ group $(n=101)$ Coefficient of correlation Coefficient of correlation

\begin{tabular}{lll}
\hline BAP & 0.29369 & 0.49270 \\
P1NP & 0.19633 & 0.48437 \\
TRACP-5b & 0.23399 & 0.40877 \\
uNTX & 0.15384 & 0.38299 \\
SCTX & 0.17631 & 0.36991 \\
\hline
\end{tabular}


Fig. 3 Percent changes of the lumbar spine BMD from the baseline at 48 weeks. Black bars indicate the ALN + ELD group; white bars indicate the ALN + VitD group. Data are represented as LS mean \pm SE. Patients were stratified into tertiles according to the baseline levels of the BTMs: a sCTX, b uNTX, c TRACP-5b, d $\mathrm{BAP}$, and $\mathbf{e} \mathrm{P} 1 \mathrm{NP}$. The number below each bar represents number of patients of each group a SCTX

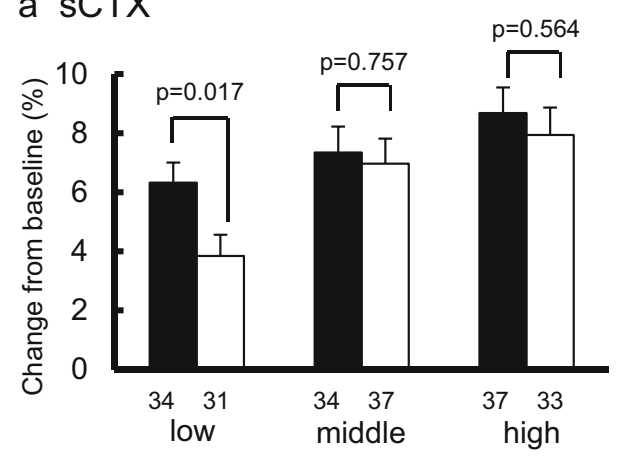

b uNTX

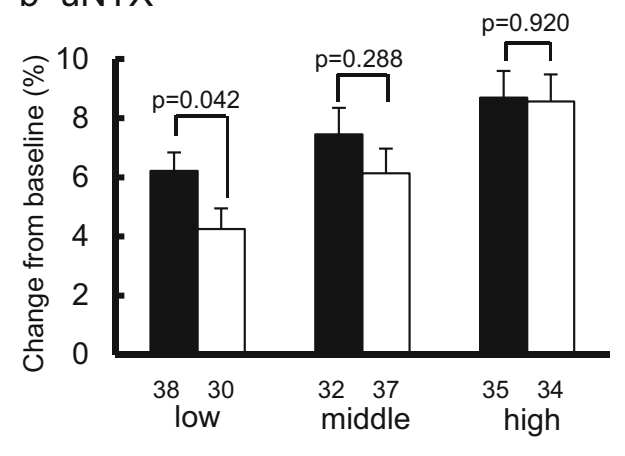

c TRACP-5b

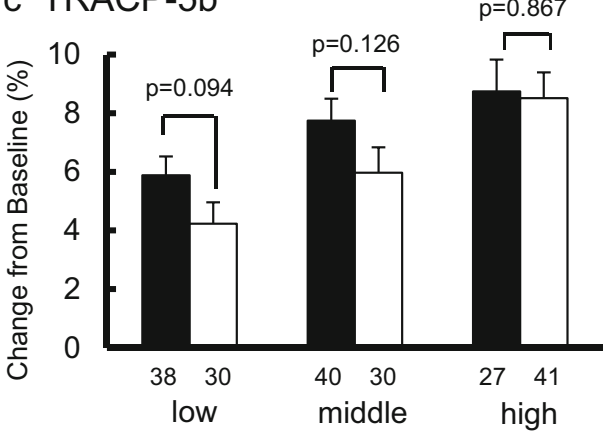

d BAP

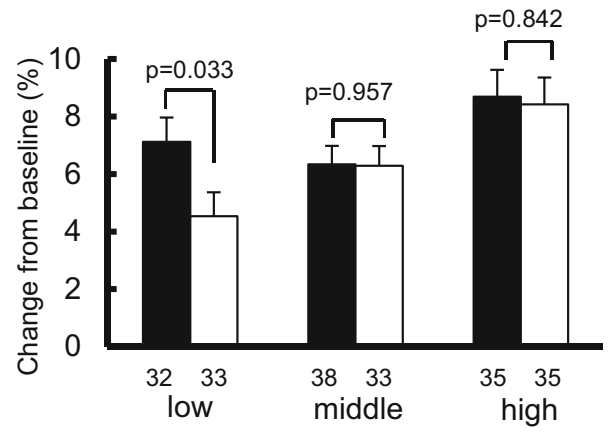

e P1NP

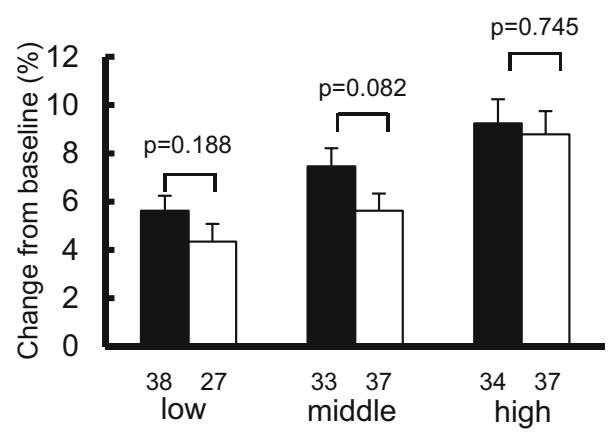

Tertile boundaries for bone turnover markers

\begin{tabular}{lccc}
\hline & Low & Middle & High \\
\hline sCTX $(\mathrm{ng} / \mathrm{mL})$ & $<0.414$ & $\geq 0.414-<0.635$ & $\geq 0.635$ \\
uNTX $(\mathrm{nmol} \mathrm{BCE} / \mathrm{mmol} \mathrm{Cr})$ & $<54.8$ & $\geq 54.8-<74.8$ & $\geq 74.8$ \\
$\operatorname{TRACP}-5 \mathrm{~b}(\mathrm{mU} / \mathrm{dL})$ & $<412$ & $\geq 412-<557$ & $\geq 557$ \\
$\operatorname{BAP}(\mu \mathrm{g} / \mathrm{L})$ & $<15.0$ & $\geq 15.0-<21.2$ & $\geq 21.2$ \\
P1NP $(\mathrm{ng} / \mathrm{mL})$ & $<41.5$ & $\geq 41.5-<62.7$ & $\geq 62.7$ \\
\hline
\end{tabular}

were significantly larger in the ALN + ELD group than those in the ALN + VitD group. The increases of the FN-BMD in patients with lower baseline values of SCTX were significantly larger in the ALN + ELD group than those in the ALN + VitD group (Fig. 4).

Patients were also stratified by the baseline serum $25(\mathrm{OH}) \mathrm{D}$ levels into two groups $(<20 \mathrm{ng} / \mathrm{mL}$ or $\geq 20 \mathrm{ng} / \mathrm{mL}$ ). There was no correlation between the percent increase of the L-BMD and the baseline serum 25(OH) D level in either group (Fig. 5a). On the other hand, the percent increase of the FN-BMD was markedly higher in the patients with serum $25(\mathrm{OH}) \mathrm{D}$ levels $<20 \mathrm{ng} / \mathrm{mL}$ in ALN + ELD group as compared to that in the ALN + VitD group (Fig. 5b).

Fracture incidences and falls

The cumulative incidence of new vertebral fractures during the 48 -week treatment period was $6.3 \%(n=7$ out of 110$)$ in the ALN + ELD group and $4.6 \%$ ( $n=5$ out of 109$)$ in the ALN + VitD group. The incidence of osteoporotic non-vertebral 
FN-BMD stratified by sCTX

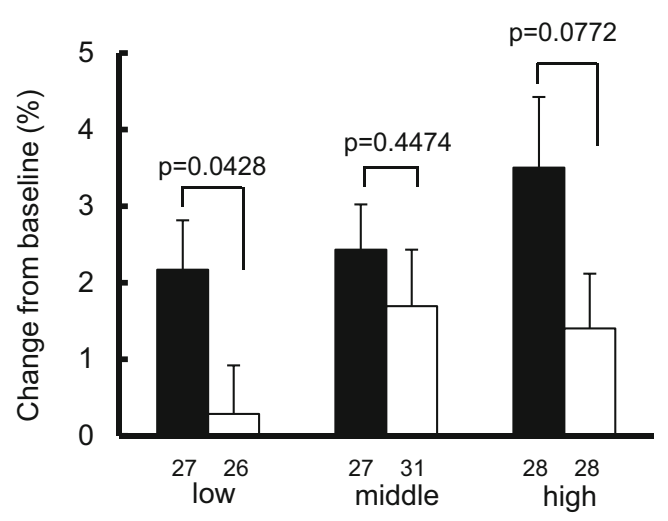

Fig. 4 Percent changes of the femoral neck BMD from the baseline at 48 weeks. Black bars indicate the ALN + ELD group; white bars indicate the ALN + VitD group. Data are represented as LS mean \pm SE. Patients were stratified into tertiles according to the baseline SCTX levels. The number below each bar represents number of patients of each group

fractures was $0.0 \%(n=0 / 110)$ in the ALN + ELD group and $4.6 \%(n=5 / 109)$ in the ALN + VitD group. The cumulative incidence of self-reported falling during the 48 -week treatment period was $24.5 \%(n=27 / 110)$ in the ALN + ELD group and $29.4 \%(n=32 / 109)$ in the ALN + VitD group, with no significant difference between the two groups.

\section{Adverse events}

The total incidence of adverse events during the study period was $86.4 \%(95 / 110,280$ events $)$ in the ALN + ELD group, and $86.2 \%(94 / 109 ; 271$ events) in the ALN + VitD group. The adverse events are listed in Table 3. No clinically meaningful changes of the laboratory data, including the blood biochemical, hematological or urine exam data were detected during the study period (not shown). No significant changes of the mean serum calcium or urinary calcium excretion levels from the baseline were observed in either the ALN + ELD group or the ALN + VitD group at any time-point during the study period (not shown). Overall, no significant differences were observed between the two groups with respect to the incidence/severity of any adverse events.

\section{Discussion}

ALN and ELD were found to exert additive anti-resorptive effects in patients with osteoporosis in this study. ALN reduces bone resorption by suppressing osteoclast activity and inducing osteoclast apoptosis via inhibition of farnesyl pyrophosphate synthetase in the cells [5]. On the other hand, it has been observed that ELD reduces the osteoclast number by suppressing the expression of RANKL in the osteoblasts [9], stimulating differentiation of preosteoblasts to osteoblasts [10] and shifting osteoclast precursor cells to the blood circulation [11]. Recent studies have unraveled that microRNAs regulate osteoclast [12] and osteoblast differentiation [13], and may be involved in the epigenetic regulation of target genes by vitamin D $[14,15]$. These apparently different anti-resorptive actions at the cellular and molecular levels of ALN and ELD may be able to work synergistically in reducing the bone turnover in men and postmenopausal women with osteoporosis.

The effect of combined treatment with ALN and ELD on the FN-BMD, namely, a $2.7 \%$ increase of the BMD over the treatment period of 48 weeks, seemed to be clinically important. In fact, the increase of the FN-BMD associated with bisphosphonate treatment plus vitamin D and calcium supplementation is reportedly minimal and usually less than $2.0 \%$ in a year, e.g., $0.4-1.8 \%$ for ALN, $0.7 \%$ for ibandronate, and $0.0 \%$ for risedronate [16-21]. However, more potent antiresorptive agents such as denosumab [22] and odanacatib [23] produced a more pronounced increase of the FN-BMD, by approximately 2.7 and $2.5 \%$, respectively, in 1 year. Thus, the efficacy of combined treatment with ALN and ELD on the

Fig. 5 Percent changes from the baseline of the a lumbar spine BMD and $\mathbf{b}$ femoral neck BMD at 48 weeks. Black bars indicate the ALN + ELD group; white bars indicate the ALN + VitD group. Data are represented as LS mean \pm SE. Patients were stratified into two groups according to the baseline serum $25(\mathrm{OH})$ D levels $(<20 \mathrm{ng} / \mathrm{mL}$ or $\geq 20 \mathrm{ng} / \mathrm{mL})$. The number below each bar represents number of patients of each group
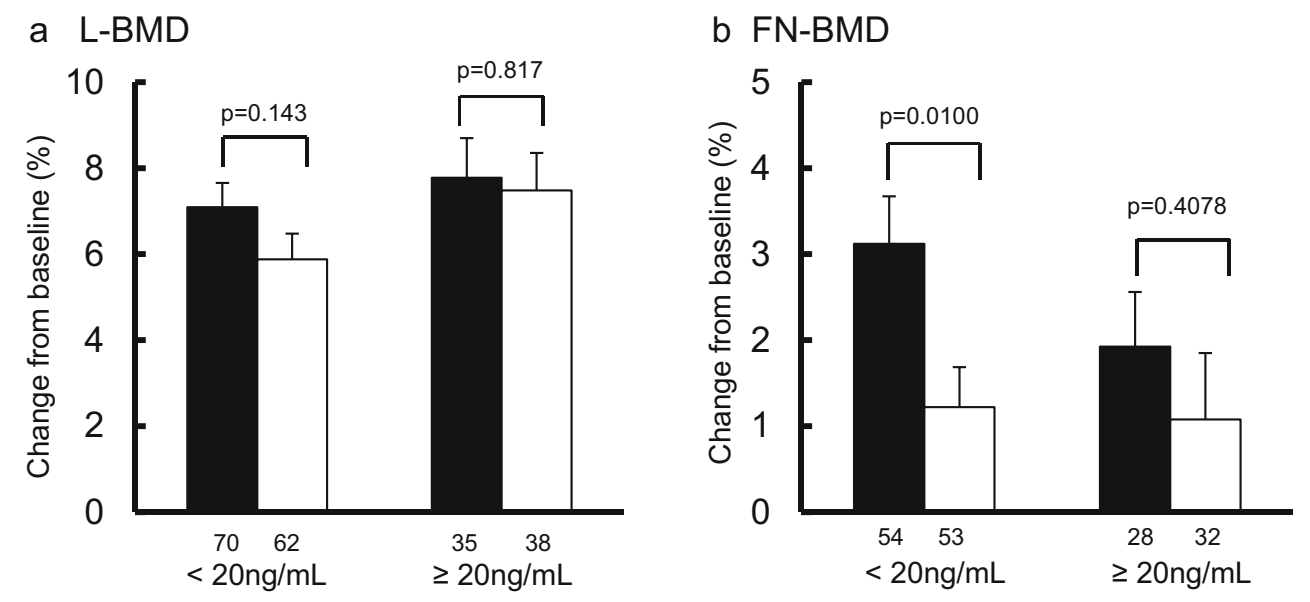
Table 3 Incidences of adverse events

\begin{tabular}{|c|c|c|}
\hline & $\begin{array}{l}\text { ALN + ELD group }(n=110) \\
N(\%)\end{array}$ & $\begin{array}{l}\text { ALN group }(n=109) \\
N(\%)\end{array}$ \\
\hline Any adverse events & $95(86.4 \%)$ & $94(86.2 \%)$ \\
\hline Infection & $50(45.5 \%)$ & $41(37.6 \%)$ \\
\hline Injury, poisoning, and procedural complications & $38(34.5 \%)$ & $43(39.4 \%)$ \\
\hline Musculoskeletal and connective tissue disorders & $33(30.0 \%)$ & $36(33.0 \%)$ \\
\hline Gastrointestinal disorders & $28(25.5 \%)$ & $29(26.6 \%)$ \\
\hline Skin and subcutaneous tissue disorders & $15(13.6 \%)$ & $18(16.5 \%)$ \\
\hline Investigations & $11(10.0 \%)$ & $5(4.6 \%)$ \\
\hline Ear and labyrinth disorders & $8(7.3 \%)$ & $1(0.9 \%)$ \\
\hline Nervous system disorders & $8(7.3 \%)$ & $7(6.4 \%)$ \\
\hline Respiratory, thoracic and mediastinal disorders & $7(6.4 \%)$ & $8(7.3 \%)$ \\
\hline Eye disorders & $5(4.5 \%)$ & $11(10.1 \%)$ \\
\hline Vascular disorders & $5(4.5 \%)$ & $2(1.8 \%)$ \\
\hline Neoplasms benign, malignant and unspecified (including cysts and polyps) & $4(3.6 \%)$ & $3(2.8 \%)$ \\
\hline Cardiac disorders & $3(2.7 \%)$ & $1(0.9 \%)$ \\
\hline Metabolism and nutrition disorders & $3(2.7 \%)$ & $4(3.7 \%)$ \\
\hline Psychiatric disorders & $2(1.8 \%)$ & $2(1.8 \%)$ \\
\hline Blood and lymphatic system disorders & $1(0.9 \%)$ & $2(1.8 \%)$ \\
\hline General disorders and administration site conditions & $1(0.9 \%)$ & $6(5.5 \%)$ \\
\hline Immune system disorders & $1(0.9 \%)$ & \\
\hline Reproductive system and breast disorders & $1(0.9 \%)$ & $1(0.9 \%)$ \\
\hline Hepatobiliary disorders & & $1(0.9 \%)$ \\
\hline Renal and urinary disorders & & $2(1.8 \%)$ \\
\hline
\end{tabular}

FN-BMD may be comparable with that of these potent antiresorptive agents, at least over a treatment period of 1 year.

The synergistic effects of ALN and ELD in reducing the bone turnover (anti-resorptive activity) could be one of the reasons to explain the efficacy of combined ALN plus ELD therapy on the BMD. The degree of reduction in the levels of the BTMs during ALN treatment has been shown to be correlated with the long-term BMD response [24-27]. Thus, enhancement of the anti-resorptive activity of ALN by the addition of ELD may contribute to the increase of the BMD. Another explanation is the potent Ca-mobilizing action of ELD. ELD has been shown to be comparable to the active form of vitamin $\mathrm{D}_{3}$, such as $1 \alpha$-hydroxyvitamin $\mathrm{D}_{3}$ or $1 \alpha$, $25(\mathrm{OH})_{2} \mathrm{D}_{3}$ [28-30], in stimulating Ca transport in the intestine. It has also been observed that ELD is more efficacious in enhancing bone mineralization than plain vitamin D3 in rodent models of vitamin D deficiency [31, 32]. Thus, the potent activity of ELD in stimulating intestinal $\mathrm{Ca}$ absorption may account for its stronger effect on the BMD, especially the FNBMD, in patients with low baseline serum $25(\mathrm{OH})$ D levels.

It has been observed that the baseline levels of the BTMs influence the increase of the L-BMD and hip BMD occurring following ALN treatment. In fact, patients with high baseline levels of the BTMs tended to show a greater percent increase of the BMD than patients with low baseline levels of the
BTMs at the start of the ALN treatment in the Fracture Intervention Trial [33]. This study also confirmed the strong correlation with the percent increase of the L-BMD in ALN + VitD group. However, combined treatment with ALN + ELD weakened the relationship. Actually, differences in percent increase values of L-BMD and F-BMD were not apparent between the high and the low tertile subgroups of BTMs in ALN + ELD group, compared to the inter-subgroup differences in ALN + VitD group. The increased potency of reducing bone turnover and intestinal $\mathrm{Ca}$ absorption by the combination of ALN and ELD may be related to the findings.

This study had several limitations. It was not a doubleblind study, and both the investigators and patients were aware of the treatment assigned to the patients. Inter-group differences in the changes of the L-BMD, which provided the basis for the power calculation in the study, were not statistically significant. The safety profile did not differ between the groups in this study, but the size of the study population and duration of the study were not large/long enough to compare the incidences of rare adverse events. This study was not powered to detect the incidences on fractures and falls between the groups either.

In conclusion, combined treatment with $35 \mathrm{mg}$ of ALN weekly and $0.75 \mu \mathrm{g}$ of ELD daily for 48 weeks was found to be more effective in reducing the BTMs in Japanese men and 
postmenopausal women with osteoporosis. The combined treatment was also found to potently increase the FN-BMD. ELD seemed to exert additive anti-resorptive activity and enhance the potent intestinal calcium absorption in patients receiving ALN monotherapy for 48 weeks.

Acknowledgments The study was jointly designed by primary investigator and the sponsor, Chugai Pharmaceutical and Taisho pharmaceutical. The sponsor had responsibility for data collection and quality control. Analyses for publication were the responsibilities of the sponsor. All authors contributed to the manuscript and approved the final version for submission. The authors would like to acknowledge the other members of the e-ADVANCED study group: Hiroo Yamane, Masahiro Shiraishi, Kunitaka Menuki, Sanshiro Hashimoto, Toshihiro Sadamatsu, Tomohiro Fukuda, Kazuki Miyazono, Hirofumi Kataoka, Masataka Gotoh, and Ohsei tsuji. The study was funded by Taisho Pharmaceutical.

Conflicts of interest AS has received consultant fee and honoraria from Astellas, Chugai and Taisho-Toyama. MI has received research grants from Chugai, and consultant fee and/or honoraria from Asahi Kasei, Astellas, Chugai, Daiichi, Ono, and Sankyo. HT has received honoraria from Asahi Kasei, Astellas, Chugai, Daiichi Sankyo, Eisai, Eli Lilly Japan, Ono, Pfizer, Taisho-Toyama, Takeda, and Teijin. SI has received consultant fee and honoraria from Asahi Kasei, Astellas, Chugai, Daiichi Sankyo, Eisai, Eli Lilly Japan, Ono, Taisho-Toyama, Takeda, and Teijin. FF has received honoraria from Stryker Japan and Takeda. HM has received consulting fee, honoraria and/or research funding from Asuka, Chugai, Eisai, Eli Lilly Japan, Fuji, Hisamitsu, Kaken, Kissei, Mochida, Nippon-shinyaku, Ono, Pfizer, Sato, Serono Japan, Taiho, and TaishoToyama. TI is an employee of Taisho. HS is an employee of Chugai. TN has received consultant fee and honoraria from Asahi Kasei, Astellas, Chugai, Daiichi Sankyo, Eli Lilly Japan, Merck, Taisho-Toyama, Teijin, and belongs to the Japan Ministry of Health, Welfare and Labor as a counselor for hospital administration and social medical insurance. TT declares no conflict of interest.

Open Access This article is distributed under the terms of the Creative Commons Attribution Noncommercial License which permits any noncommercial use, distribution, and reproduction in any medium, provided the original author(s) and the source are credited.

\section{References}

1. Miyamoto K, Murayama E, Ochi K, Watanabe H, Kubodera N (1993) Synthetic studies of vitamin D analogues. XIV. Synthesis and calcium regulating activity of vitamin D3 analogues bearing a hydroxyalkoxy group at the 2 beta-position. Chem Pharm Bull (Tokyo) 41:1111-1113

2. Matsumoto T, Miki T, Hagino H, Sugimoto T, Okamoto S, Hirota T, Tanigawara Y, Hayashi Y, Fukunaga M, Shiraki M, Nakamura T (2005) A new active vitamin D, ED-71, increases bone mass in osteoporotic patients under vitamin D supplementation: a randomized, double-blind, placebo-controlled clinical trial. J Clin Endocrinol Metab 90:5031-5036

3. Matsumoto T, Ito M, Hayashi Y, Hirota T, Tanigawara Y, Sone T, Fukunaga M, Shiraki M, Nakamura T (2011) A new active vitamin D3 analog, eldecalcitol, prevents the risk of osteoporotic fractures-a randomized, active comparator, double-blind study. Bone 49:605612. doi:10.1016/j.bone.2011.07.011
4. NIH Consensus Development Panel on Osteoporosis Prevention, Diagnosis, and Therapy (2001) Osteoporosis prevention, diagnosis, and therapy. JAMA 285:785-795

5. Russell RG, Xia Z, Dunford JE, Oppermann U, Kwaasi A, Hulley PA, Kavanagh KL, Triffitt JT, Lundy MW, Phipps RJ, Barnett BL, Coxon FP, Rogers MJ, Watts NB, Ebetino FH (2007) Bisphosphonates: an update on mechanisms of action and how these relate to clinical efficacy. Ann N Y Acad Sci 1117:209-257

6. Orimo H, Hayashi Y, Fukunaga M, Sone T, Fujiwara S, Shiraki M, Kushida K, Miyamoto S, Soen S, Nishimura J, Oh-Hashi Y, Hosoi T, Gorai I, Tanaka H, Igai T, Kishimoto H, Osteoporosis Diagnostic Criteria Review Committee: Japanese Society for Bone and Mineral Research (2001) Diagnostic criteria for primary osteoporosis: year 2000 revision. J Bone Miner Metab 19:331-337

7. Mori S, Soen S, Hagino H, Nakano T, Ito M, Fujiwara S, Kato Y, Tokuhashi Y, Togawa D, Endo N, Sawaguchi T, Committee for Vertebral Fracture Evaluation (2013) Justification criteria for vertebral fractures: year 2012 revision. J Bone Miner Metab 31:258-261. doi:10.1007/s00774-013-0441-1

8. Genant HK, Jergas M, Palermo L, Nevitt M, Valentin RS, Black D, Cummings SR (1996) Comparison of semiquantitative visual and quantitative morphometric assessment of prevalent and incident vertebral fractures in osteoporosis. J Bone Miner Res 11:984-996

9. Harada S, Mizoguchi T, Kobayashi Y, Nakamichi Y, Takeda S, Sakai S, Takahashi F, Saito H, Yasuda H, Udagawa N, Suda T, Takahashi N (2012) Daily administration of eldecalcitol (ED-71), an active vitamin $\mathrm{D}$ analog, increases bone mineral density by suppressing RANKL expression in mouse trabecular bone. J Bone Miner Res 27:461-473. doi:10.1002/jbmr.555

10. de Freitas PH, Hasegawa T, Takeda S, Sasaki M, Tabata C, Oda K, Li M, Saito H, Amizuka N (2011) Eldecalcitol, a second-generation vitamin $\mathrm{D}$ analog, drives bone minimodeling and reduces osteoclastic number in trabecular bone of ovariectomized rats. Bone 49:335-342. doi:10.1016/j.bone.2011.05.022

11. Kikuta J, Kawamura S, Okiji F, Shirazaki M, Sakai S, Saito H, Ishii M (2013) Sphingosine-1-phosphate-mediated osteoclast precursor monocyte migration is a critical point of control in antiboneresorptive action of active vitamin D. Proc Natl Acad Sci U S A 110:7009-7013. doi:10.1073/pnas.1218799110

12. Krzeszinski JY, Wei W, Huynh H, Jin Z, Wang X, Chang TC, Xie XJ, He L, Mangala LS, Lopez-Berestein G, Sood AK, Mendell JT, Wan Y (2014) miR-34a blocks osteoporosis and bone metastasis by inhibiting osteoclastogenesis and Tgif2. Nature 512:431-435. doi: 10.1038 /nature 13375

13. Li H, Xie H, Liu W, Hu R, Huang B, Tan YF, Xu K, Sheng ZF, Zhou HD, Wu XP, Luo XH (2009) A novel microRNA targeting HDAC5 regulates osteoblast differentiation in mice and contributes to primary osteoporosis in humans. J Clin Invest 119:3666-3677. doi:10.1172/ JCI39832

14. Lisse TS, Adams JS, Hewison M (2013) Vitamin D and microRNAs in bone. Crit Rev Eukaryot Gene Expr 23:195-214

15. Campbell MJ (2014) Vitamin D and the RNA transcriptome: more than mRNA regulation. 5:1-13. doi: 10.3389/fphys.2014.00181

16. Scott LJ (2014) Denosumab: a review of its use in postmenopausal women with osteoporosis. Drugs Aging 31:555-576. doi:10.1007/ s40266-014-0191-3

17. Shiraki M, Kushida K, Fukunaga M, Kishimoto H, Taga M, Nakamura T, Kaneda K, Minaguchi H, Inoue T, Morii H, Tomita A, Yamamoto K, Nagata Y, Nakashima M, Orimo H (1999) A double-masked multicenter comparative study between alendronate and alfacalcidol in Japanese patients with osteoporosis. The Alendronate Phase III Osteoporosis Treatment Research Group. Osteoporos Int 10:183-192

18. Kendler DL, Roux C, Benhamou CL, Brown JP, Lillestol M, Siddhanti S, Man HS, San Martin J, Bone HG (2010) Effects of denosumab on bone mineral density and bone turnover in 
postmenopausal women transitioning from alendronate therapy. $\mathrm{J}$ Bone Miner Res 25:72-81. doi:10.1359/jbmr.090716

19. Brown JP, Prince RL, Deal C, Recker RR, Kiel DP, de Gregorio LH, Hadji P, Hofbauer LC, Alvaro-Gracia JM, Wang H, Austin M, Wagman RB, Newmark R, Libanati C, San Martin J, Bone HG (2009) Comparison of the effect of denosumab and alendronate on BMD and biochemical markers of bone turnover in postmenopausal women with low bone mass: a randomized, blinded, phase 3 trial. J Bone Miner Res 24:153-161. doi:10.1359/jbmr.080901

20. Recknor C, Czerwinski E, Bone HG, Bonnick SL, Binkley N, Palacios S, Moffett A, Siddhanti S, Ferreira I, Ghelani P, Wagman RB, Hall JW, Bolognese MA, Benhamou CL (2013) Denosumab compared with ibandronate in postmenopausal women previously treated with bisphosphonate therapy: a randomized open-label trial. Obstet Gynecol 121: 1291-1299. doi:10.1097/AOG.0b013e318291718c

21. Roux C, Hofbauer LC, Ho PR, Wark JD, Zillikens MC, FahrleitnerPammer A, Hawkins F, Micaelo M, Minisola S, Papaioannou N, Stone M, Ferreira I, Siddhanti S, Wagman RB, Brown JP (2014) Denosumab compared with risedronate in postmenopausal women suboptimally adherent to alendronate therapy: efficacy and safety results from a randomized open-label study. Bone 58:48-54. doi: 10.1016/j.bone.2013.10.006

22. Nakamura T, Matsumoto T, Sugimoto T, Hosoi T, Miki T, Gorai I, Yoshikawa H, Tanaka Y, Tanaka S, Sone T, Nakano T, Ito M, Matsui $\mathrm{S}$, Yoneda T, Takami H, Watanabe K, Osakabe T, Shiraki M, Fukunaga M (2014) Clinical trials express: fracture risk reduction with denosumab in Japanese postmenopausal women and men with osteoporosis: denosumab fracture intervention randomized placebo controlled trial (DIRECT). J Clin Endocrinol Metab 99:2599-2607. doi:10.1210/jc.2013-4175

23. Nakamura T, Shiraki M, Fukunaga M, Tomomitsu T, Santora AC, Tsai R, Fujimoto G, Nakagomi M, Tsubouchi H, Rosenberg E, Uchida S (2014) Effect of the cathepsin K inhibitor odanacatib administered once weekly on bone mineral density in Japanese patients with osteoporosis - a double-blind, randomized, dosefinding study. Osteoporos Int 25:367-376. doi:10.1007/s00198013-2398-2

24. Greenspan SL, Parker RA, Ferguson L, Rosen HN, MaitlandRamsey L, Karpf DB (1998) Early changes in biochemical markers of bone turnover predict the long-term response to alendronate therapy in representative elderly women: a randomized clinical trial. J Bone Miner Res 13:1431-1438

25. Ravn P, Clemmesen B, Christiansen C (1999) Biochemical markers can predict the response in bone mass during alendronate treatment in early postmenopausal women. Alendronate Osteoporosis Prevention Study Group. Bone 24:237-244

26. Nenonen A, Cheng S, Ivaska KK, Alatalo SL, Lehtimäki T, SchmidtGayk H, Uusi-Rasi K, Heinonen A, Kannus P, Sievänen H, Vuori I, Väänänen HK, Halleen JM (2005) Serum TRACP 5b is a useful marker for monitoring alendronate treatment: comparison with other markers of bone turnover. J Bone Miner Res 20:1804-1812

27. Iwamoto J, Takeda T, Sato Y, Uzawa M (2005) Early changes in urinary cross-linked $\mathrm{N}$-terminal telopeptides of type I collagen level correlate with 1-year response of lumbar bone mineral density to alendronate in postmenopausal Japanese women with osteoporosis. J Bone Miner Metab 23:238-242

28. Okano T, Tsugawa N, Masuda S, Takeuchi A, Kobayashi T, Takita Y, Nishii Y (1989) Regulatory activities of 2 beta-(3-hydroxypropoxy)1 alpha, 25-dihydroxyvitamin D3, a novel synthetic vitamin D3 derivative, on calcium metabolism. Biochem Biophys Res Commun 163:1444-1449

29. Saito H, Harada S (2014) Eldecalcitol replaces endogenous calcitriol but does not fully compensate for its action in vivo. J Steroid Biochem Mol Biol 144:189-196. doi:10.1016/j.jsbmb.2013.11.013

30. Matsumoto T, Takano T, Yamakido S, Takahashi F, Tsuji N (2010) Comparison of the effects of eldecalcitol and alfacalcidol on bone and calcium metabolism. J Steroid Biochem Mol Biol 121:261-264. doi: 10.1016/j.jsbmb.2010.03.035

31. Chugai Pharmaceutical Co., Ltd. [Common Technical Document: Eldecalcitol 2.6.2.2.1.6]. http://www.info.pmda.go.jp/shinyaku/ P201100025/index.html. Japanese

32. Ochi H (1998) 2 $\beta$-(3-Hydroxypropoxy) $1 \alpha$,25-Dihydroxyvitamin $\mathrm{D}$ (3) (ED-71), increases bone mass by stimulating bone formation in hypophosphatemic mice. J Okayama Med Assoc 110:31-37, Japanese

33. Bauer DC, Garnero P, Hochberg MC, Santora A, Delmas P, Ewing SK, Black DM; Fracture Intervention Research Group (2006) Pretreatment levels of bone turnover and the antifracture efficacy of alendronate: the fracture intervention trial. J Bone Miner Res 21:292-299 